\title{
Load Balancing Algorithm Exploiting Overlay Techniques
}

\author{
Anton Dort-Golts, Olga Simonina \\ Networks Department, State University of Telecommunications, Saint-Petersburg, Russia \\ dortgolts@gmail.com, simonina@bk.ru
}

\begin{abstract}
In the article we propose network traffic load balancing mechanism, based on the identification of a single network- or transport-layer traffic flows. The principal idea of proposed algorithm is a dynamical observation of the distribution router links current load, with such routers combined in special overlay network. For the purpose of load balancing some single flows after reaching load threshold could be relocated to alternative routes. Proposed algorithm is able to make selective flows relocation taking into account QoS demands of each single flow.
\end{abstract}

Keywords — load balancing, P2P, overlay networks, traffic engineering, QoS

\section{INTRODUCTION}

Urgency of this research is caused by irregularity of traffic loads aroused on the access layer by ISP's different segments users. It is known, that traffic load correlations with time of the day, subscriber`s age, area of interests and familiarity of up-to-date Internet-technologies could be observed [1] Techniques, that are employed today to estimate the bandwidth of distribution-level router links, commonly use reducing coefficients, i.e. expect channel utilization not higher than some value [2]. But they don't take into account impact of traffic burstiness, irregular distribution of the subscribers at the given moment and L3-VPN usage caused to ensure QoS demands. Current load balancing mechanisms utilize routing protocols capabilities and take into account channel bandwidth or /and DSCP field value of single IP-packets. But when we try to introduce NGN services there is a necessity of certain flows relocation occurs. This is caused by channel switching emulation on the network layer and representing traffic of different services, especially streaming ones (online-video, IPtelephony, conferencing) as a variable bitrate channels. Proposed algorithm employs load balancing mechanisms, based on the overlay networks and permits to redirect flows selectively, taking into account QoS demands of each certain flow. It is supposed that such algorithm could be used on any layers performing routing functions.

\section{RELATED WORK}

Attempts to efficiently redistribute packet network traffic has been making since the appearance of routing in ARPANet. This research area has its own name - Traffic Engineering (TE). First trend of traffic load optimization was MPLS-TE [3], allowing to balance traffic on the channel-layer (L2).
Network-layer support of TE methods for the popular routing protocols, such as OSPF and IS-IS, was proposed as an extension of the protocols [4]-[5]. There are also hybrid solutions, such as [6], trying to combine and harmonize routing on both levels: MLPS and OSPF. All this mentioned approaches are used to be called "offline"-methods, and their key drawback is preliminary alternative routes computation based on the long-term averaged network load information. The consequence of such an approach is impossibility to adequately react to the momentary traffic bursts, which cannot be predicted, raised by BGP reroutes, flash crowds, attacks etc.

To eliminate these drawbacks there were some "online"methods developed [7]-[8], that mostly are centralized or use oracle. Also we should mention decentralized "online"mechanisms, for instance TeXCP [9] - distributed TEprotocol, making it possible to react to the changing traffic load in real-time. Other examples of such an approach to be mentioned are self-configuring TE scheme SculpTE [10] and DACoRM [11] - adaptive resource management system for intra-domain traffic engineering.

\section{III.OVERVIEW}

There are two typical approaches to connect up access networks and the core backbone network presented on the figure 1. We will further call scheme presented on the fig.1a first-type or tree-like hierarchical network, and on the fig. $1 \mathrm{~b}-$ second-type or mesh-network. It is obvious also that first-type network could be considered as a particular case of secondtype network with one fixed router, connected to all other routers and possible lack of some links between latters. However, considering prevalence of first-type network it is useful to examine load balancing system operation for both models separately.

In this article we propose algorithm, equalizing traffic load on the trunk links of distribution routers. Hence, for the case presented on fig.1a loads of links between distribution layer routers (DR) and core layer router (CR) should be equalized, while in the fig. $1 \mathrm{~b}$ case algorithm should evenly distribute traffic load between all the routes connecting DRs.

As a soft criterion for load balancing effectiveness estimation we propose following inequality.

$$
\begin{aligned}
{\left[\max _{i}\left\{\lambda_{i}^{(\text {before })}\right\}\right.} & \left.-\min _{i}\left\{\lambda_{i}^{(\text {before })}\right\}\right] \\
& \geq\left[\max _{i}\left\{\lambda_{i}^{(\text {after })}\right\}-\min _{i}\left\{\lambda_{i}^{(\text {after })}\right\}\right]
\end{aligned}
$$




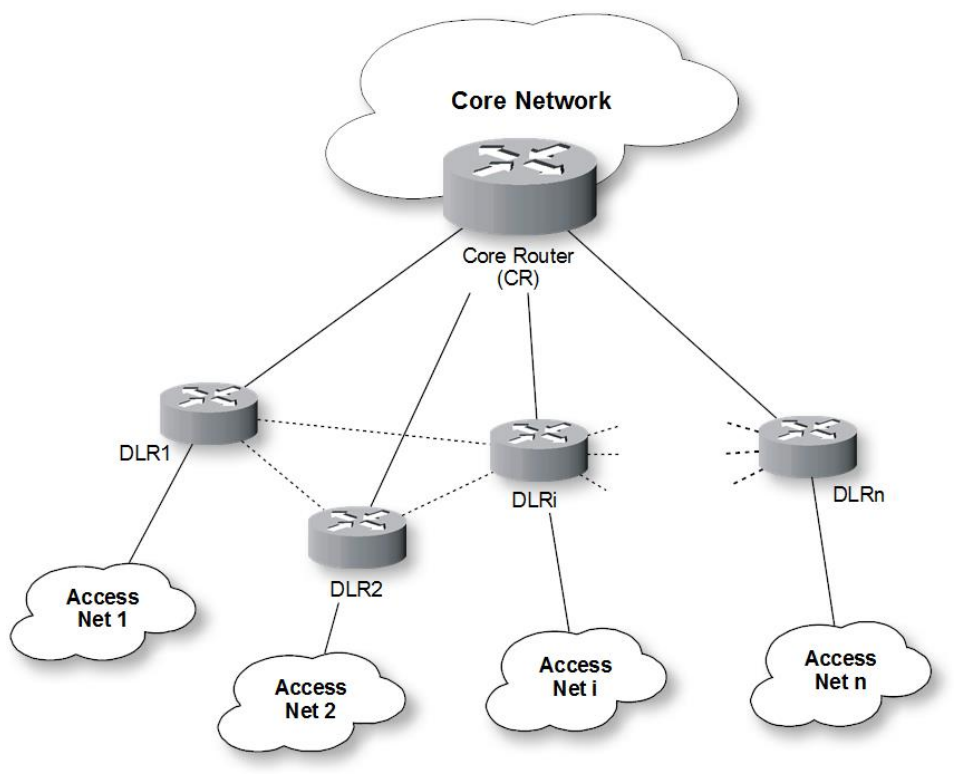

a)

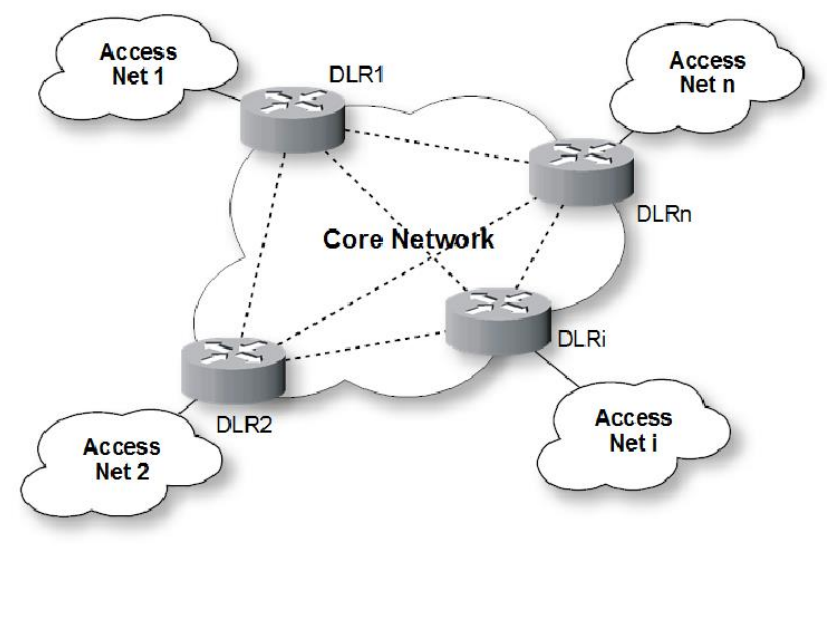

b)

Figure 1. Two typical distribution network models

Here $\lambda_{i}^{\left({ }^{(b e f o r e}\right)}$ and $\lambda_{i}^{\left({ }^{(a f t e r}\right)}-$ traffic intensity in each $i$-th trunk before loading procedure and after it, accordingly.

The basic idea of the proposed algorithm is opportunity to choose some non-optimal route for the traffic that is not so sensitive for QoS parameters such as delay and delay variation, for example, file sharing.

Due to this decision it becomes possible to equalize trunk links load solely by means of network layer. Also, algorithm could be used to ensure QoS requirements of different types of clients: with different bandwidth demands, different link costs etc.

For implementing such balancing we propose to divide set of all routers into several subsets: groups. Traffic load balancing procedure takes place only on routers participating in the same group. Watching over the group state and routers operation suggested to be implemented with special overlay network - we call it $L B O$ (Load Balancing Overlay). This overlay network is fully decentralized flat topology structure, which allows to dynamically coordinate operation of collaborating routers in the same group and also to make "hot plugging/unplugging" of the routers without necessity of some manual administration.

\section{IV.NETWORK MODELS AND PARAMETERS}

In the article we are concentrated on the network layer L3. Thus lower layers could be based on any technologies and protocols and use any underlying physical network implementation. Application area of algorithm include ISP`s networks providing NGN services and using IP-routing, and also corporate communication networks, linking its separate sites together through the transport network of some detached service provider.

\section{A. First-type network (tree-like)}

Distribution routers participating in one group organize decentralized peer-to-peer overlay network. In terms of this network further we will call these routers as nodes.

For convenience to describe some distributed network parameters we use here matrices and vectors by analogy with widely used traffic matrices.

Topology of tree-like network including $n$ routers is presented at fig.1a. Let's describe capacity of channels connecting DRs with the core router as a vector $\left(c_{1}^{(\text {out })}, c_{2}^{\text {(out })}, \ldots, c_{n}^{(\text {out })}\right)$. Connections between DRs could be described with following matrix.

$$
C^{(i n t)}=\left[\begin{array}{ccc}
0 & \cdots & c_{1 n}^{(i n t)} \\
\vdots & \ddots & \vdots \\
c_{n 1}^{(i n t)} & \cdots & 0
\end{array}\right],
$$

Here $c_{i j}^{(i n t)}$ - capacity of the communication channel between nodes $i$ and $j$.

It should be noted, that capacity estimation task is charged exclusively to corresponding nodes. Capacities could be assumed equal to the capabilities of corresponding interfaces multiplied by some correction factor, or be directly measured when new nodes join the overlay. 
Each $i$-th node observes following parameters:

- Traffic intensity in the link connecting this node to core router $\lambda_{i}^{\text {(out) }}$.

- Traffic intensity in the internal links connecting different DRs, which can be represented as a matrix.

$$
\lambda^{(i n t)}=\left[\begin{array}{ccc}
0 & \cdots & \lambda_{1 n}^{(i n t)} \\
\vdots & \ddots & \vdots \\
\lambda_{n 1}^{(i n t)} & \cdots & 0
\end{array}\right],
$$

Here $\lambda_{i j}^{(i n t)}$ - traffic load flowing in the direction from $i$-th node towards $j$-th one. Generally speaking such matrix is not symmetrical.

As soon as traffic load in some channel reaches its threshold value, load smoothing procedure corrects this unbalance. Threshold loads are initially specified and could be adjusted later. These loads for channels connecting DRs and $\mathrm{CR}$ could be described as a vector: $\lambda_{C R}{ }^{(t r)}=\left(\lambda_{1}^{(t r)}, \lambda_{2}^{(t r)}, \ldots, \lambda_{n}^{(t r)}\right)$, and for internal channels as following matrix.

$$
\lambda_{\text {int }}{ }^{(t r)}=\left[\begin{array}{ccc}
0 & \cdots & \lambda_{1 n}^{(t r)} \\
\vdots & \ddots & \vdots \\
\lambda_{n 1}^{(t r)} & \cdots & 0
\end{array}\right]
$$

In the elementary case threshold value linearly depends on capacity of corresponding channel, for example $\lambda_{i}^{(t r)}=$ $k_{t r} c_{i}^{(o u t)}, k_{t r}=0.7$.

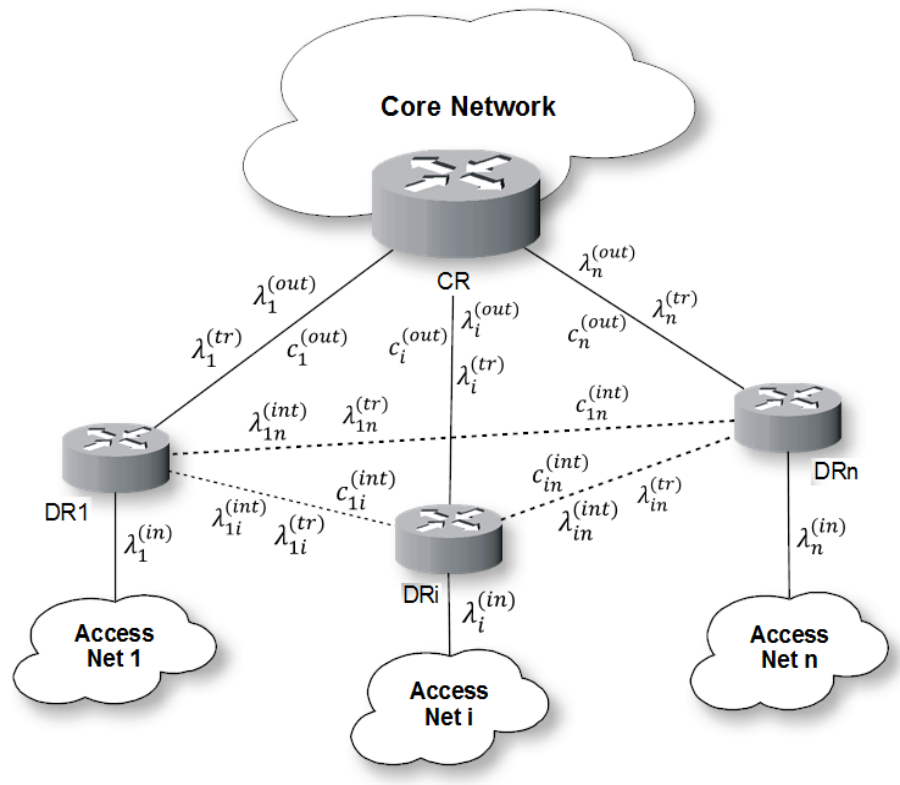

Figure 2. Tree-like network model

Described network model is represented on figure 2 .

\section{B. Second-type network (mesh)}

In contrast to tree-like network model here channels between nodes are not distinguished. We assume that such network is fully-connected, therefore we can represent all above parameters as matrices with no zero elements except the main diagonal.

Symmetrical matrix of channel capacities:

$$
C^{(\text {out })}=\left[\begin{array}{ccc}
0 & \cdots & c_{1 n}^{(o u t)} \\
\vdots & \ddots & \vdots \\
c_{n 1}^{(o u t)} & \cdots & 0
\end{array}\right]
$$

Here $c_{i j}^{(o u t)}$ - capacity of the channel connecting nodes $i$ and $j$.

Threshold values matrix:

$$
\lambda^{(t r)}=\left[\begin{array}{ccc}
0 & \cdots & \lambda_{1 n}^{(t r)} \\
\vdots & \ddots & \vdots \\
\lambda_{n 1}^{(t r)} & \cdots & 0
\end{array}\right]
$$

Traffic loads between nodes:

$$
\lambda^{(\text {out })}=\left[\begin{array}{ccc}
0 & \cdots & \lambda_{1 n}^{(\text {out })} \\
\vdots & \ddots & \vdots \\
\lambda_{n 1}^{(o u t)} & \cdots & 0
\end{array}\right]
$$

Here $\lambda_{i j}^{(o u t)}-$ traffic load flowing in the direction from $i$-th node towards $j$-th one.

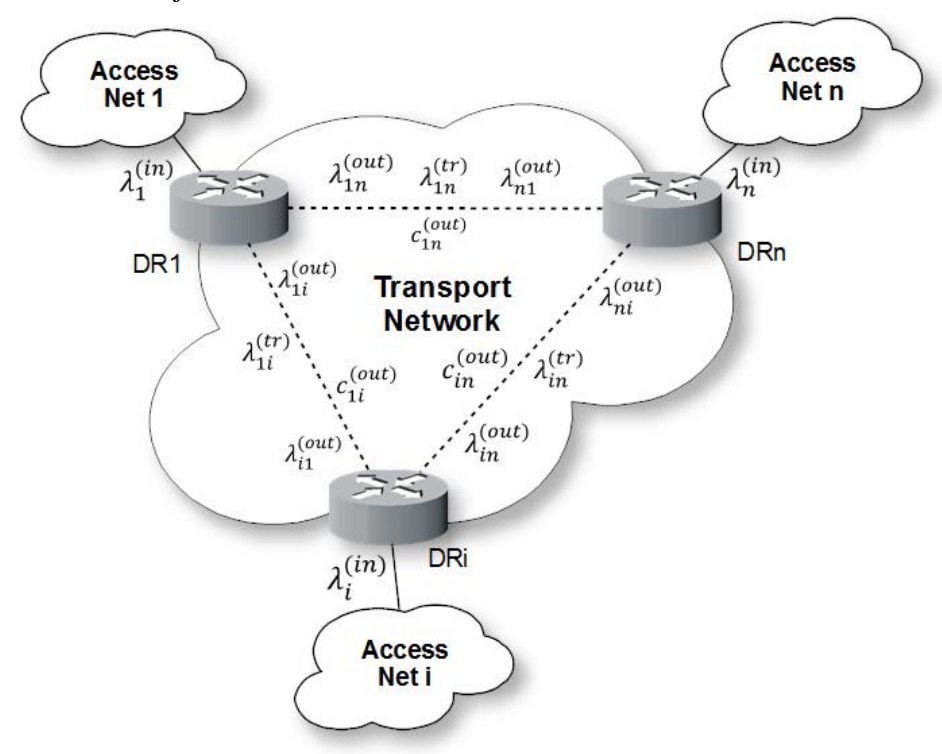

Figure 3. Mesh-network model

Traffic exchange intensity between routers participating in load balance overlay and corresponding access networks in both models is described with following vector: $\lambda^{(i n)}=$ $\left(\lambda_{1}^{(i n)}, \lambda_{2}^{(i n)}, \ldots, \lambda_{n}^{(i n)}\right)$.

\section{NETWORK MANAGEMENT}

On the initial stage of LBO operation participating nodes join the special self-organized overlay network, which allows 
TABLE 1. EXAMPLE OF NEIGHBOR DATABASE IN TREE-LIKE BALANCING NETWORK

\begin{tabular}{|c|c|c|c|c|c|c|c|c|c|c|}
\hline$i$ & IP-address & $\lambda_{i}^{(o u t)}$ & $\lambda_{i}^{(t r)}$ & $c_{i}^{(\text {out })}$ & $\lambda_{i 1}^{(i n t)}$ & $\lambda_{i 1}^{(t r)}$ & $c_{i}^{(o u t)}$ & $\lambda_{i n}^{(i n t)}$ & $\lambda_{i n}^{(t r)}$ & $c_{i}^{\text {(out })}$ \\
\hline 1 & 172.10 .8 .3 & 351 & 700 & 1000 & 0 & 0 & 0 & 215 & 700 & 1000 \\
\hline 2 & 172.10 .6 .7 & 726 & 700 & 1000 & 672 & 700 & 1000 & 611 & 700 & 1000 \\
\hline $\mathrm{n}$ & 172.10 .65 .1 & 548 & 850 & 1000 & 354 & 850 & 1000 & 0 & 0 & 0 \\
\hline
\end{tabular}

nodes to exchange data and maintains self-acting node join/leave procedure. Algorithms of building such network are beyond this article and will be examined in future publications.

\section{A. Tree-like network}

All nodes participating in LBO should be aware of current values of above parameters to be able to redistribute flows effectively. For this purpose each node maintains neighbor database, and simplified example of such database is presented in table 1.

For keeping its database up-to date each node periodically, once at $t_{\text {update }}$ seconds, sends UPDATE messages to all nodes, whose addresses are stored in the database. Each UPDATE message contains timestamp, current parameters of channel between present node and CR: $\lambda_{i}^{(\text {out })}, \lambda_{i}^{(t r)}, c_{i}^{(\text {out })}$ vectors $\left(\lambda_{i 1}^{(i n t)}, \lambda_{i 2}^{(i n t)}, \ldots, \lambda_{i n}^{(i n t)}\right),\left(\lambda_{i 1}^{(t r)}, \lambda_{i 2}^{(t r)}, \ldots, \lambda_{i n}^{(t r)}\right)$ and $\left(c_{i 1}^{\text {(out })}, c_{i 2}^{\text {(out })}, \ldots, c_{i n}^{\text {out }}\right)$, showing load of channels connecting present node with all other nodes, except $\mathrm{CR}$, and set of its neighbor`s IP-addresses. When some node receives UPDATE message from one of its neighbors, it updates corresponding values in its own neighbor database.

If during the period $k_{\text {fail }} t_{\text {update }}$ (here $k_{\text {fail }}$ - factor) some node doesn't receive any UPDATE message from one of its neighbors, such neighbor considered to be inactive and should removed from database, and all connections remapped to this node should be relocated on other nodes.

\section{B. Mesh-network}

Nodes in mesh-network have more generalized neighbor database, as it is shown in table 2 .

TABLE 2. EXAMPLE OF NEIGHBOR DATABASE IN MESH-NETWORK

\begin{tabular}{|c|c|c|c|c|c|c|c|}
\hline$i$ & IP-address & $\lambda_{i 1}^{(\text {out })}$ & $\lambda_{i 1}^{(t r)}$ & $c_{i 1}^{(\text {out })}$ & $\lambda_{\text {in }}^{(\text {out })}$ & $\lambda_{i n}^{(t r)}$ & $c_{\text {in }}^{\text {(out) }}$ \\
\hline 1 & 172.10 .8 .3 & 0 & 0 & 0 & 351 & 700 & 1000 \\
\hline 2 & 172.10 .6 .7 & 672 & 700 & 1000 & 611 & 700 & 1000 \\
\hline $\mathrm{n}$ & 172.10 .5 .1 & 548 & 800 & 1000 & 0 & 0 & 0 \\
\hline
\end{tabular}

ope

Traffic generated by LBO, containing $n$ nodes in both cases could be estimated not higher than $O\left(n^{2}\right)$ messages, each message size - about $O(n)$.

\section{VI.LOAD CONTROL}

Irrespective of LBO network type, each node permanently observes the load of its trunk channels (i.e. exclusively interfaces that reside in the overlay). Load inspection performs periodically, $k_{\text {probe }}$ times during the $t_{\text {update }}$ interval. Obtained results are averaged, and further written into own neighbor database and transmitted to other nodes in UPDATE messages.

Performing this periodical check is necessary for detection some channels failures or overload (congestion) being a result of increased traffic transmission intensity of one or several connections. If failure or congestion is detected, node randomly chooses several flows among all the connections, that are allowed for remapping (see "load smoothing procedure"), making their way through this node, and relocate this flows to other nodes. Amount of relocated flows is proportional to the overload of the channel.

Channel overload may be detected by two ways:

- Measurements on corresponding interfaces. This method is easy to implement, but it doesn`t give any information about possible congestion in bottlenecks of physical intermediate channels, connecting two nodes.

- Probing. Each node periodically, once at $\frac{t_{\text {update }}}{k_{\text {probe }}}$ seconds, sends small ECHO packets to all its neighbors, obtained from neighbor database. Measuring the period since probe packet was transmitted until the acknowledgment for it is received (i.e. round-trip time) we could estimate available bandwidth of channel connecting any pair of nodes.

\section{LOAD SMOOTHING}

Procedure called load smoothing could be divided into two parts: flows distribution and remapping. Load smoothing operates independently of routing protocols deployed in network and requires no changes in their implementations.

It should be noted, that load smoothing procedure could be applied only to the flows, which has no strong requirements for delay and jitter (delay variation) parameters. Also it is 


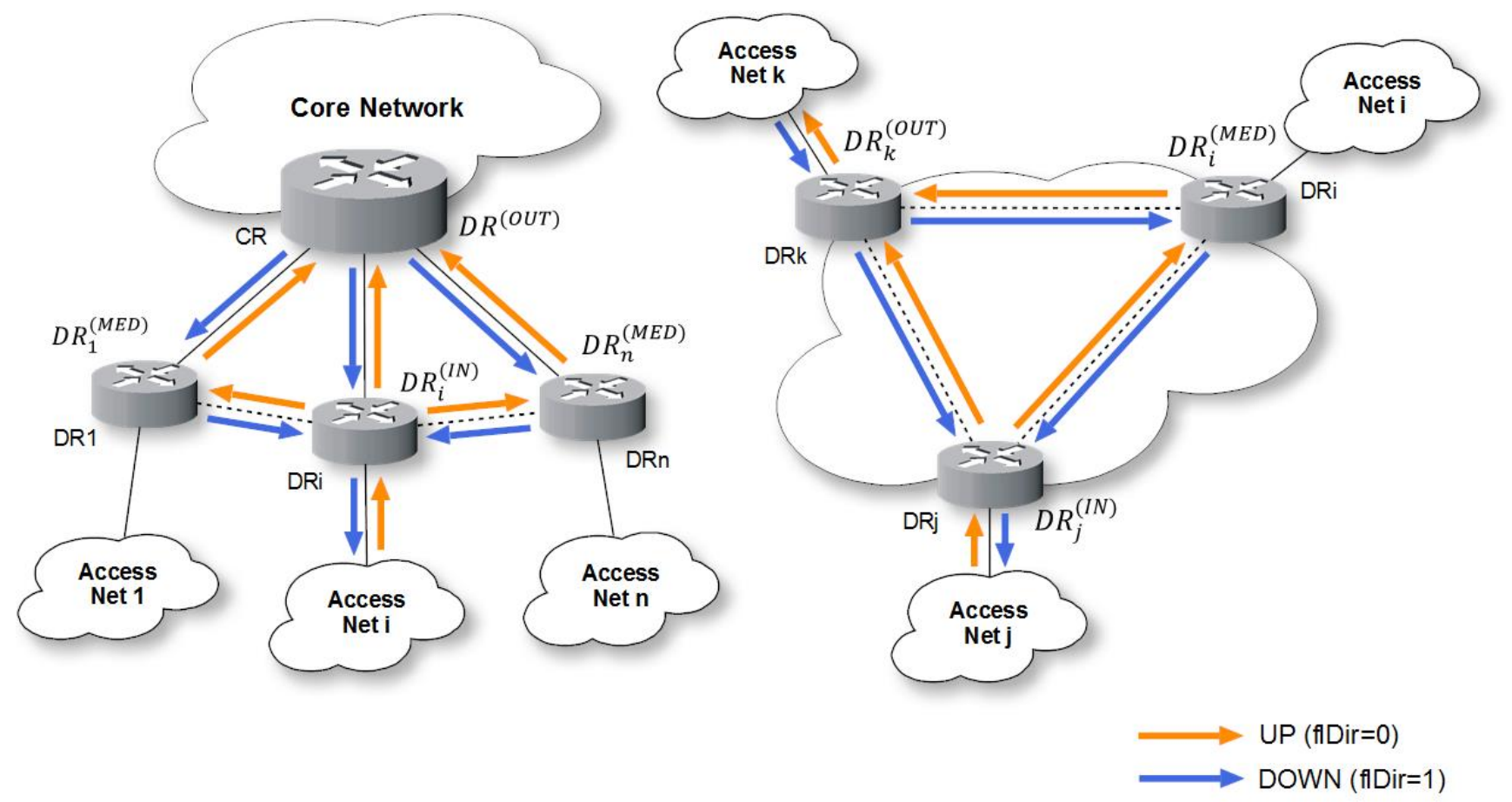

Figure 4. Flow directions

possible to implement routing mechanism, which can take into account different traffic classes.

Following parameters pair unambiguously identifies all traffic flows in LBO:

- $\left[s r c_{i p}: s r c_{\text {port }} ; d s t_{i p}: d s t_{\text {port }}\right]$, if we need to balance open traffic, containing transport layer protocols (e.g., UDP/TCP);

- $\left[s r c_{i p} ; d s t_{i p}\right]$, if subscriber`s traffic is transmitted, being encapsulated in packets of some tunneling protocol (e.g., any VPN-protocol).

Along with flow identification we also should detect its direction. As soon as first packet of some new flow, trying to establish connection, gets to the node of $\mathrm{LBO}$, its direction, i.e. order of sockets or IP-addresses pair, considered being UP (node marks it flag flDir=0). Consequently, when packets of this flow later will get back into present node, node will recognize its direction as DOWN (marked with flag flDir=1).

Every packet making its way through one of LBO`s nodes should be preliminary checked by balancing subsystem before it will get into routing subsystem. Such check procedure is explained in paragraph "Flow remapping".

While doing flow identification at the same time we should perform traffic type verification with the purpose of detection flows, which could be sensitive to delays and jitter. We may employ different techniques: from checking DSCP flags in IPpackets to statistical behavioural patterns and DPI L7. Flows critical to specified parameters are not relocatable, and marked by additional flag flRemapPermit $=0$, applied to all packets of this flow. Specified flag is set to the whole flow in the first phase of connection establishment on the grounds of packet analysis, and further all packets classified as relevant to some flow, should be treated according to this flow`s flRemapPermit value.

\section{A. Amount of remapped flows estimation}

Principal idea of proposed load balancing algorithm is that each node participating in LBO in order to avoid overloading of its own channels, should relocate traffic flows, crowded on certain route, to other relatively free channels, connecting nodes. Since increasing the number of additional hops implies in significant increase of computational complexity and network delay, it makes sense to restrict set of alternative routes: only routes which has one additional hop (compared to main shortest path) could be used for remapping. Hence, shortest route through the LBO should pass two nodes, and all alternative paths - three nodes.

Thus, usage of non-optimal routes allows us to reach more even traffic load distribution over the network.

Each node participating in LBO periodically (once at $t_{\text {update }}$ seconds) computes number of flows to remap: these flows should either be relocated from overloaded route to alternative ones or, in the case of vacant resources in shortest route, previously remapped flows should be returned. For the simplicity let's have a good look at the case, when the only criteria of remapping necessity is the relative overload (and underload too) of shortest route. Let's assume that all shortest routes are obtained by independent routing subsystem.

Total number of flows, passing certain shortest path between two nodes of the same LBO, which should be remapped to alternative routes, can be estimated by following expression. 


$$
m=N f_{R E S}\left(\lambda^{(\text {out })}\right) ;|m| \leq N
$$

Here $\lambda^{\text {(out) }}-$ current load of shortest path, $N$ - total number of flows, which are not remapped yet, $f_{R E S}$ - estimation function for residual resources of certain shortest path. In this article for simplicity we'll consider only linear form of $f_{R E S}$ dependence on residual resources.

$$
f_{R E S}\left(\lambda^{\text {out }}\right)=\frac{\lambda^{(\text {out })}-\lambda^{(t r)}}{c^{(\text {out })}-\lambda^{(t r)}}
$$

In the specified case load balancing system behaviour will be symmetrical around the threshold load value (see fig. 5). Negative number of flows informs us of possibility to return $m$ or less previously remapped flows to the shortest path.

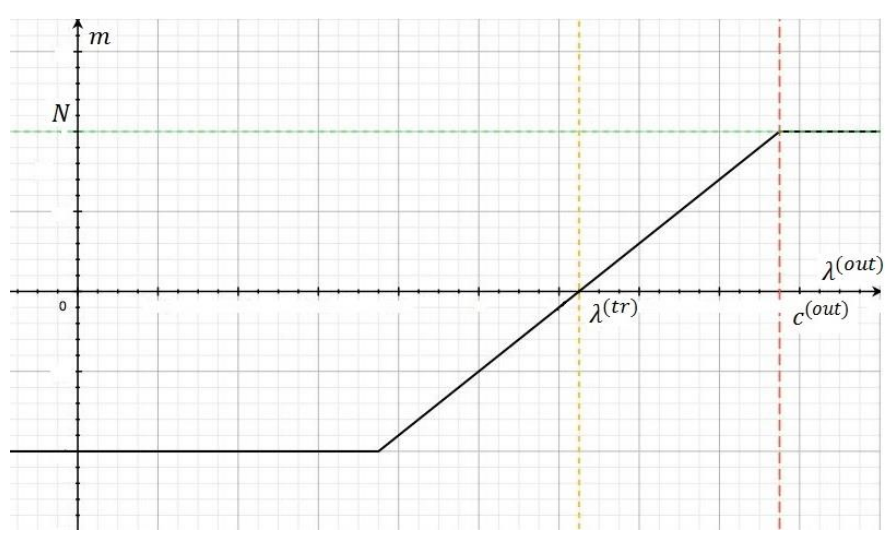

Figure 5. Amount of flows to remap vs. current load of the shortest path

One of the well-known problems of load balancing systems is oscillation of routes, especially when shortest path load is around the threshold value. In order to remove this obstacle we propose here additional function, which defines remapping probability of some channel depending on closeness of load value to threshold. Example of such oscillation avoidance (OA) probability function may look like following one (see fig. 6).

$$
\begin{aligned}
& p\left(\lambda^{(\text {out })}\right)= \\
& =\left\{\begin{array}{c}
1, \lambda^{(\text {out })}<\lambda^{(\text {tr })}-a \\
1-\left(1-\frac{\lambda^{(\text {out })}-\lambda^{(\text {tr })}}{a}\right)^{k_{O A}}, \lambda^{(\text {tr })}-a \leq \lambda^{(\text {out })}<\lambda^{(\text {tr })} \\
1-\left(1+\frac{\lambda^{(t r)}-\lambda^{(\text {out })}}{a}\right)^{k_{O A}}, \lambda^{(\text {tr })} \leq \lambda^{(\text {out })}<\lambda^{(\text {tr })}+a \\
1, \quad \lambda^{(\text {out })} \geq \lambda^{(\text {tr })}+a
\end{array}\right.
\end{aligned}
$$

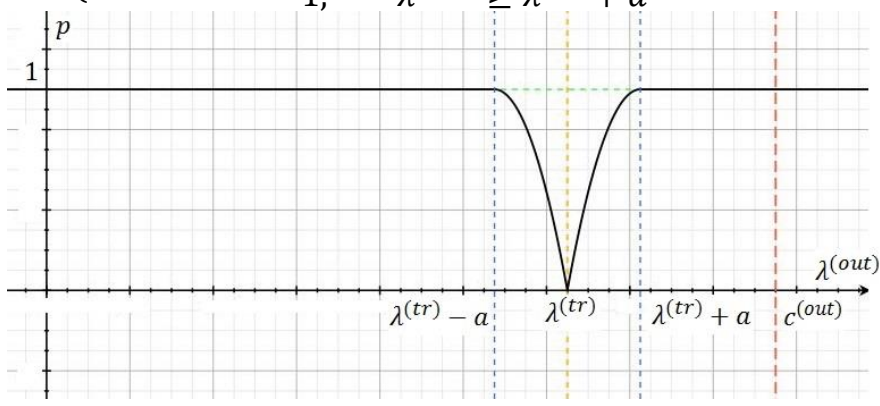

Figure 6. Probability of flow remapping
Here parameter $a$ specifies area around the threshold value, where probability will be less than 1 , and $k_{O A}$ - non-linearity factor, defining probability decrease rate as it approaches the threshold.

Now, having oscillation avoidance function, aimed to reduce route oscillations, total number of flows, which should be remapped to alternative routes, can be expressed as:

$$
m=N f_{R E S}\left(\lambda^{(\text {out })}\right) p\left(\lambda^{(\text {out })}\right) ;|m| \leq N
$$

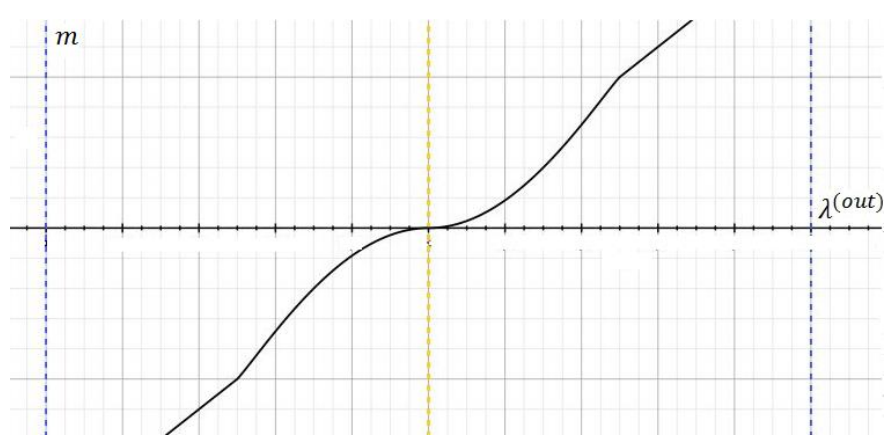

Figure 7. Influence of proposed oscillation avoidance function on amount of remapped flows

The influence of proposed additional OA probability function on total amount of flows to be remapped is depicted on figure 7. As can be seen from the graph, number of remapped flows remains small enough in some area around the threshold.

Adjusting $a$ and $k_{O A}$ variables we could achieve desired system behavior. This issue lies beyond the article and may be researched in future.

\section{B. Flows distribution}

Obtained, as it was described in the previous paragraph, number of flows to remap should then be distributed among other nodes, which have unused reserves of residual channel bandwidth. In the elementary case such distribution may be directly proportional to the residual bandwidth of each roundabout alternative route.

1) Tree-like network model: Introducing linear estimation function depending on residual unused bandwidth of shortest route, passing through $i$-th node.

$$
\begin{gathered}
g_{i}=\min \left\{\frac{\lambda_{i}^{(t r)}-\lambda_{i}^{(\text {out })}}{\lambda_{i}^{(t r)}} ; \frac{\lambda_{j i}^{(t r)}-\max \left\{\lambda_{j i}^{(i n t)} ; \lambda_{i j}^{(i n t)}\right\}}{\lambda_{j i}^{(t r)}}\right\} ; \\
\forall i=1 \ldots n \mid \lambda_{i}^{(\text {out })}<\lambda_{i}^{(t r)}, \max \left\{\lambda_{j i}^{(\text {int })} ; \lambda_{i j}^{(\text {int })}\right\}<\lambda_{j i}^{(t r)}, i \neq j .
\end{gathered}
$$

For the specified network topology amount of flows between nodes $j$ and CR to remap on the route passing through $i$-th node could be expressed as:

$$
m_{i}=N f_{R E S}\left(\lambda_{j}^{o u t}\right) p\left(\lambda_{j}^{\text {out }}\right) \frac{g_{i}}{\sum_{k=1}^{n} g_{k}}
$$


TABLE 3. EXAMPLE OF FLOW-CHART DATABASE

\begin{tabular}{|c|c|c|c|c|c|c|}
\hline $\begin{array}{c}\text { Flow } \\
\text { ID }\end{array}$ & $\begin{array}{c}\left(\mathrm{src}_{\mathrm{IP}}: \mathrm{src}_{\text {port }} ;\right. \\
\left.\mathrm{dst}_{\mathrm{IP}}: \mathrm{dst}_{\mathrm{port}}\right)\end{array}$ & flRemapPermit & isRemapped & $D R_{I P \text { addr }}^{(I N)}$ & $D R_{I P \text { addr }}^{(\text {MED })}$ & $D R_{I P \text { addr }}^{(\text {OUT })}$ \\
\hline 1 & $\begin{array}{c}(198.16 .11 .4: 332 ; \\
8.42 .7 .16: 7478)\end{array}$ & 1 & 1 & 172.10 .5 .8 & 172.10 .16 .25 & 172.12 .4 .9 \\
\hline 2 & $\begin{array}{c}(198.16 .11 .4: 8080 ; \\
71.4 .12 .1: 80)\end{array}$ & 0 & - & - & - & - \\
\hline 3 & $\begin{array}{c}(198.16 .131 .4: 9948 ; \\
144.1 .12 .8: 2343)\end{array}$ & 1 & 0 & - & - & - \\
\end{tabular}

In the case of negative $m_{i}$, obtained number of previously remapped flows could be returned from each $i$-th roundabout route to the $j$-th node's shortest path.

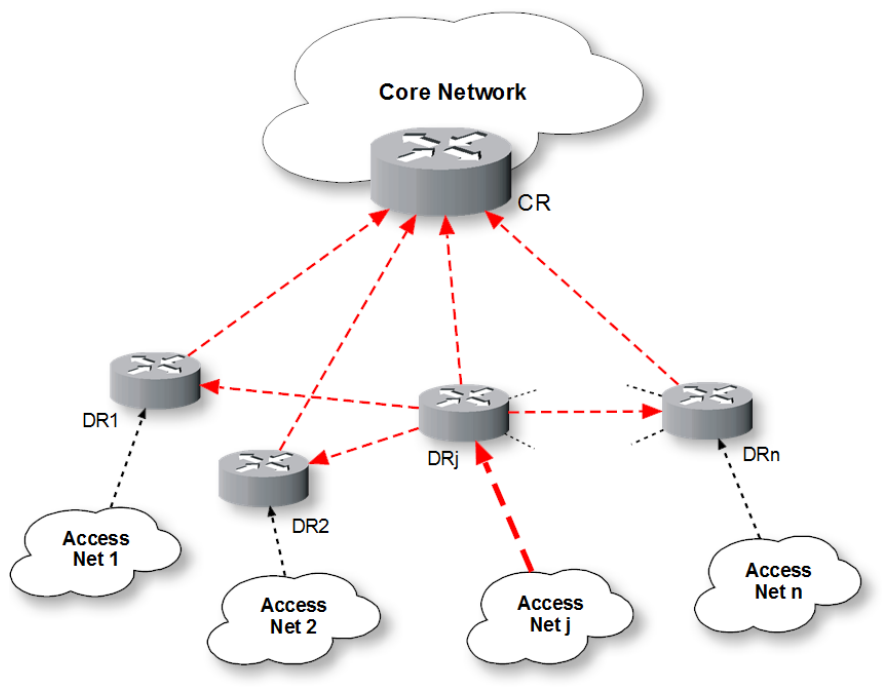

Figure 8. Flows redistribution in tree-like network

2) Mesh-network: Expressions for the mesh-network case are similar to ones examined above, but slightly more generalized. Residual bandwidth estimation function:

$$
\begin{gathered}
g_{i}=\min \left\{\begin{array}{l}
\frac{\lambda_{j i}^{(t r)}-\max \left\{\lambda_{j i}^{(\text {out })} ; \lambda_{i j}^{(\text {out })}\right\}}{\lambda_{j i}^{(t r)}} ; \\
\frac{\lambda_{i k}^{(t r)}-\max \left\{\lambda_{i k}^{(\text {out })} ; \lambda_{k i}^{(o u t)}\right\}}{\lambda_{i k}^{(t r)}}
\end{array}\right\} ; \\
\forall i=1 \ldots n \mid \max \left\{\lambda_{j i}^{(\text {out })} ; \lambda_{i j}^{(\text {out })}\right\}<\lambda_{j i}^{(t r)}, \\
\max \left\{\lambda_{i k}^{(\text {out })} ; \lambda_{k i}^{(o u t)}\right\}<\lambda_{i k}^{(t r)}, i \neq j, k .
\end{gathered}
$$

Number of flows to remap, utilizing OA function:

$$
m_{i}=N f_{R E S}\left(\lambda_{j k}^{\text {out }}\right) p\left(\lambda_{j k}^{(\text {out })}\right) \frac{g_{i}}{\sum_{k=1}^{n} g_{k}}
$$

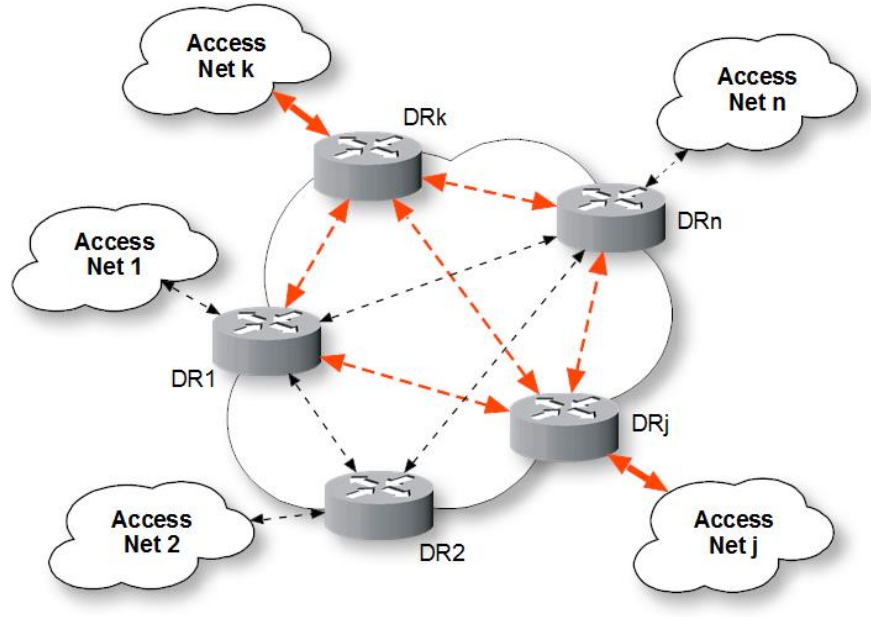

Figure 9. Flows redistribution in mesh-network

\section{Remapping}

As amount of flows to be remapped for each of available neighbours is obtained, node should relocate these flows from overloaded shortest route to corresponding roundabout paths.

Each node in the LBO maintains its own flow-chart database, in which information about all the flows passing through this node is stored. Simplified example of flow-chart presented in table 3

Node initiating remapping of one or several flows (nodeinitiator, further denoted as $D R_{j}^{(I N)}$ ) sends REMAP message to chosen node-mediator $\left(D R_{i}^{(M E D)}\right)$. This message contains set of data strings, looking similar to following one:

$\left\{\operatorname{src}_{I P}: \operatorname{src}_{\text {port }} ; d s t_{I P}: d s t_{\text {port }} ; D R_{I P}^{(I N)}\right.$ address $\left.; D R_{I P \text { address }}^{(M E D)} ; D R_{I P \text { address }}^{(O U T)}\right\}$,

Each data string contains information about single flow to be remapped and IP-addresses of nodes in the roundaboutroute chain: node-initiator $\left(D R_{j}^{(I N)}\right)$, node-mediator $\left(D R_{i}^{(M E D)}\right)$ and node-recipient $\left(D R_{k}^{(O U T)}\right)$. All this information nodeinitiator $D R^{(I N)}$ also stores in its own flow-chart (see table 3) with flag isRemapped $=1$. Necessity to restrict number of additional hops in alternative routes implies marking and detachment of those flows, which already have been 
previously remapped from another node, to prevent recurring procedure. For the purpose of such detachment is used special flag isRemapped. Additional flag flRemapPermit shows if certain flow could be remapped or not according to its QoS demands. For example, traffic of such RT-applications as IPtelephony, conferencing etc. should not be remapped to avoid undesired and/or unacceptable delays.

Any node in LBO received REMAP message, containing one of its interfaces IP-address in the $D R^{(M E D)}$ field, becomes mediator for this one or several flows, adds information obtained from the message to its own flow-chart and sends acknowledgement to initiator, notifying the latter about successful procedure. After adding information about new remapped flows into its flow-chart, mediator forms and sends REMAP messages to corresponding recipients. Each noderecipient $D R^{(O U T)}$, in it`s turn, adds information obtained from message in its flow-chart and sends acknowledgement to mediator.

In the case of tree-like network, there will be sole noderecipient - core layer router $(\mathrm{CR})$, which also resides in the overlay.

Each packet received by LBO`s node initially gets into proposed balancing subsystem, where it passes inspection: whether packets of this flow already been observed on current node or it's a brand new connection. If flow-chart contains no information about captured flow, this flow should be analyzed, as it was explained above, and corresponding entry should be added to flow-chart. Otherwise, if flow-chart has relevant entry, and the flow wasn`t remapped (i.e. it passes LBO with shortest route), captured packet gets into independent routing subsystem. If flow-chart contains relevant entry and flow was remapped, there are following possible balancing subsystem actions to be applied to the packet (actually, to all packets of certain flow):

- $\quad$ Packet received by $D R_{j}^{(I N)}$, flDir=0. Packet avoids routing subsystem and is forwarded immediately to $D R_{i}^{(M E D)}$, which IP-address could be obtained from flow-chart.

- Packet received by $D R_{j}^{(I N)}$, flDir=1. Packet gets into routing subsystem and processed as usual.

- Packet received by $D R_{i}^{(M E D)}$, flDir=0. Packet avoids routing subsystem and is forwarded immediately to $D R_{k}^{(O U T)}$, which IP-address could be obtained from flow-chart.

- Packet received by $D R_{i}^{(M E D)}$, flDir=1. Packet avoids routing subsystem and is forwarded immediately to $D R_{j}^{(I N)}$, which IP-address could be obtained from flow-chart.

- $\quad$ Packet received by $D R_{k}^{(O U T)}$, flDir=0. Packet gets into routing subsystem and processed as usual.

- Packet received by $D R_{k}^{(O U T)}$, flDir=1. Packet avoids routing subsystem and is forwarded immediately to $D R_{i}^{(M E D)}$, which IP-address could be obtained from flow-chart.
At the same time it doesn't matter, if the load balancing procedure is applied to already established connection, or a new one: after changing corresponding information in flowcharts of all nodes participating in remapping or returning previously remapped flow, certain flow will automatically change the way it goes through LBO.

\section{CONCLUSIONS}

Proposed load balancing algorithm allows redistributing traffic flows to improve QoS in the ISP network. Initially, algorithm estimates unused channel resources by observing current traffic loads, and then computes possible amount of traffic flows to be remapped from overloaded routes. Proposed algorithm has two modifications to choose, depending on employed network topology, and implements only remapping mechanism. Classification of flows by types and remapping possibility without any detriment to QoS performance considered being a distinct research problem, lying beyond the area of present article and will be analyzed in future publications.

\section{REFERENCES}

[1] Sheluhin O. I., Smolskiy S. M., Osin A. V. Self-Similar Processes in Telecommunications. - John Wiley \& Sons, Ltd, 2007.

[2] Semenov Y., Pyattaev V., Chung C. The Distributed Service Network architecture approach for Russian networks planning. Advanced Communication Technology (ICACT), 2012 14th International Conference on. - IEEE, 2012.

[3] D.Mitra and K. G. Ramakrishna. A Case Study of Multiservice Multipriority Traffic Engineering Design. In GLOBECOM, 1999.

[4] B. Fortz and M. Thorup. Optimizing OSPF Weights in a Changing World. In IEEE JSAC, 2002.

[5] B. Fortz and M. Thorup. Robust Optimization of OSPF/IS-IS Weights In INOC, 2003.

[6] M. Zhang, B. Liu and B. Zhang. Multi-commodity flow traffic engineering with hybrid MPLS/OSPF routing. In Proceedings of the 28th IEEE conference on Global telecommunications (GLOBECOM'09), NJ, USA, 2009.

[7] J. Burns, T. Ott, A. Krzesinski, and K. Müller. Path selection and bandwidth allocation in MPLS networks. Perform. Eval., no. April 2002, pp. 1-25, 2003.

[8] A. Elwalid, C. Jin, S. Low, and I. Widjaja, "MATE: MPLS adaptive traffic engineering," INFOCOM, 2001.

[9] S. Kandula, D. Katabi, B. Davie, and A. Charny. Walking the tightrope: Responsive yet stable traffic engineering. ACM SIGCOMM, 2005

[10] S. Sundaresan, C. Lumezanu. Autonomous traffic engineering with self-configuring topologies. ACM SIGCOMM, 2010.

[11] D. Tuncer, M. Charalambides. Towards decentralized and adaptive network resource management. Serv. Management., 2011.

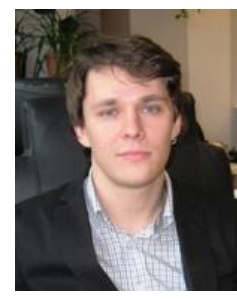

Anton Dort-Golts. Received the Specialist degree in networks and telecommunication systems from State University of Telecommunication (SUT), St. Petersburg, Russia, in 2009. At present time he`s a PhD student at Networks Department, SUT, SaintPetersburg, Russia. Currently works as an Assistant at Networks Department, SUT, SaintPetersburg, Russia. Research interests include P2P networks, overlays, statistical traffic characteristics. 


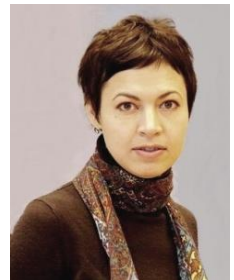

Olga Simonina received the $\mathrm{PhD}$ degree in networks and telecommunication systems from

State University of Telecommunication, St. Petersburg, Russia, in 2005. In 2000-2003 worked at Department of Information Transmission, State University of Telecommunication as an Assistant Professor, since 2003 she has been working at Networks
Department, State University of Telecommunication, as an Assistant Professor. In 2006 became an Associate Professor. Her current research interests cover quality of service, multiservice networks and overlay networks. She is an ITU-T expert (ID 04908). 